\title{
Socialización cultural y racial en las familias españolas adoptivas transraciales
}

\author{
Cultural and Racial Socialization \\ in Spanish Transracial Adoptive Families
}

\author{
María José Rodríguez-Jaume (D) https://orcid.org/0000-0002-1668-174X \\ Universidad de Alicante, España,mj.rodriguez@ua.es
}

María José González-Río (D) https://orcid.org/0000-0003-3828-2720

Universidad de Alicante,España,mj.gonzalez@ua.es

Recepción:

21/05/2018

Aprobación:

08/01/2019

\begin{abstract}
The article analyzes the particular contexts in which families that adopted children from other countries and racial groups negotiate the challenges posed by the "transracial adoption paradox": the contradictions in daily social interaction that stem from the dual social position of the children vis-à-vis their host/adopting society. While enjoying the privileges associated with living in families belonging to the racial and ethnic hegemonic group, these children are, at the same time, potentially vulnerable to the kind of discrimination and social stigmatization endured by racial minorities. The data have been extracted from the "Adoptive families and their lifestyles" survey (2012) and are based on a questionnaire answered by 230 Spanish interracial families who adopted children abroad. The results show how adoptive parents tend to put in practice socialization patterns close to assimilation/acculturation into the mainstream culture, while reproducing at the same time the ideology of colorblind racism.
\end{abstract}

Key words: transracial adoptions, colorblind racism, cultural and racial socialization, racial hierarchy, transracial family.

Resumen: El artículo aborda el estudio del proceso de socialización culturaly racial con el que las familias que adoptan a sus hijas e hijos en el extranjero afrontan la paradoja transracial. Esta describe los contextos de interacción cotidianos en los que su prole deberá negociar las contradicciones inherentes que devienen de disfrutar los privilegios asociados a vivir en un hogar que participa del tipo racial y étnico hegemónico, al tiempo que se es objeto de discriminación racial y estigmatización social, pues sus diferencias fenotípicas hacen que sea identificada como una minoría. Los datos proceden de la encuesta "Las familias adoptivas y sus estilos de vida" (2012), cumplimentada por 230 familias españolas que adoptaron en el extranjero. Los resultados describen cómo las madres y padres adoptivos transraciales despliegan patrones de socialización próximos a la asimilación cultural o aculturación en la cultura mayoritaria, reproduciendo la ideología del racismo daltónico.

Palabras clave: adopciones transraciales, racismo daltónico, socialización cultural y racial, jerarquía racial, familia transracial. 


\section{Introducción}

\section{Los límites sociales en la jerarquía racial: aportaciones desde la sociología de las adopciones ${ }^{1}$}

El fenómeno de la adopción interracial o transracial ${ }^{2}$, contemplado como "la práctica de colocar a un niño en una familia de una raza ${ }^{3}$ o grupo étnico que difiere de la familia biológica del niño" (Park, 2012: 480), desafía la norma de la homogeneidad racial familiar y plantea interrogantes relativas al papel de la institución en el proceso de socialización de sus hijas e hijos. A través de este proceso, las personas interiorizan las normas sociales, desarrollan las habilidades y las competencias necesarias para la interacción social, asumen la cultura de su entorno y desarrollan su identidad. Generalmente, en la niñez, la socialización acontece entre padres e hijos que comparten la misma raza y/o grupo étnico. En este contexto, son las propias familias y la comunidad quienes participan no solo como agentes de socialización sino también como grupo de referencia y refugio.

Sin embargo, el proceso de socialización entre las familias adoptivas transraciales aparece con tres elementos diferenciados: entre progenitores y

1 La investigación que se presenta ha sido realizada en el marco del proyecto "El (baby) boom de las adopciones internacionales en España. Una investigación sociológica sobre las familias adoptivas y sus estilos de vida" (2012) (I+D+I-2008-2011), financiado por el Ministerio de Economía y Competitividad de España.

2 En el estudio de las adopciones, los calificativos "internacionales" y "transnacionales" se suelen aplicar de forma indistinta: el primero prevalece en enfoques demográficos, mientras que el segundo se viene usando cuando se busca subrayar las dimensiones raciales, étnicas o culturales del fenómeno. A su vez, los términos familia adoptiva "transracial" o "interracial" remiten a las familias en las que los progenitores establecen lazos de parentesco con niñas y niños de origen racial y/o étnico distinto.

3 El reconocimiento por parte de la comunidad científica de que el constructo "raza" no tiene validez científica para explicar las diferencias exogrupos, unido a la extensión en sociedades democráticas de los valores de individualismo, libertad e igualdad, ha propiciado la emergencia de, siguiendo a Balibar (1991: 37), un "racismo sin razas" que sustentaría los prejuicios raciales en las diferencias culturales y no, como lo hacía el racismo tradicional, en las biológicas. No obstante, las diferencias fenotípicas - estigmas de la raza-continúan siendo utilizadas socialmente para distinguir y clasificar en: una posición económica (racismo de clase), cultura y nacionalidad (racismo cultural) y religión (racismo religioso). En este artículo centramos nuestro interés en el "racismo daltónico" o "racismo ciego al color" [color-blind racism], extensamente aplicado en el estudio de las familias adoptivas interraciales. 
prole se aprecian diferencias fenotípicas que actuarán como estigmas de la raza; las hijas e hijos viven experiencias contradictorias, ya que, en la sociedad de destino, son adscritos a minorías raciales y/o étnicas. Mientras tanto, en el ámbito familiar, incluso entre ellos mismos, son percibidos y tratados como si fueran miembros de la cultura mayoritaria debido a su adopción en una familia blanca (paradoja transracial); y el "modelo de ruptura" que la práctica adoptiva ha impuesto con la familia y comunidad de origen (Yngvesson, 2000: 171) restringe el acceso de las niñas y niños adoptados en el extranjero a los recursos que habitualmente participan en el proceso de socialización.

La investigación que aborda el proceso de socialización cultural y racial ${ }^{4}$ en familias adoptivas transraciales es un área de estudio sociológico emergente, que ofrece una visión crítica del modo en que las familias abordan diversos aspectos de la denominada paradoja transracial a la cual se enfrentan los niños de minorías raciales y étnicas que son adoptados por padres blancos (Lee, 2003: 711). Este proceso en las familias adoptivas transraciales implica el desarrollo, a lo largo de la vida de las niñas y los niños, de competencias biculturales. Esto es, siguiendo a Thomas y Tessler (2007: 1192), se socializarán tanto en la cultura dominante (país de destino) como en la de nacimiento (país de origen). El aprendizaje dual de los valores culturales, creencias y comportamientos les permitirá desarrollar tanto competencias biculturales como habilidades para afrontar posibles episodios de racismo y discriminación en contextos de interacción social (Lee, 2003: 717-718).

Adicionalmente, el estudio de las familias adoptivas transraciales ha despertado el interés entre los estudiosos de los límites sociales en la jerarquía racial, fundamentalmente en Estados Unidos. Ishizawa et al. (2006), a partir del análisis del Censo de Estados Unidos del año 2000, sugieren que

4 En España, las cuestiones raciales no generan el interés social que sí ocupa en países como Estados Unidos, Reino Unido o México, dado que es un tema hacia el que la sociedad española ha interiorizado la indeseabilidad social de cualquier manifestación de racismo o xenofobia. Sin embargo, su ausencia en el debate público no implica que la raza, como categoría socialmente construida, al igual que el género y la clase social, no sostenga los órdenes sociales. Siguiendo a Bonilla-Silva (1999; Bonilla-Silva et al., 2004), la raza se asigna externamente, se conecta a las relaciones de poder y jerarquías, y estratifica a la población otorgando privilegios (inconscientes) a quienes están adscritos al grupo cultural y racial hegemónico y, en consecuencia, discrimina al Otro. La indeseabilidad social del término raza motiva que habitualmente sea reemplazado por el de cultura o etnia (autoafirmación del carácter distintivo ligado a la creación de un sentido de comunidad). En España, la "adopción interracial" no se nombra como tal ni en la legislación específica, tampoco en los procesos administrativos que la desarrollan, ni en los documentos informativos de carácter divulgativo (San Román, 2013: 226), siendo reemplazado por el término de cultura. 
la percepción de los futuros padres sobre el significado de la raza condiciona la elección del país de procedencia de sus hijas e hijos, al tiempo que contribuye a la estratificación racial histórica que sitúa a la población blanca sobre la negra. El predominio del flujo asiático de adopciones hasta el final de la primera década del 2000 ha desencadenado un nuevo enfoque en la sociología de la etnicidad y la identidad.

Shiao et al. (2004: 8), al analizar las causas por las que la población norteamericana prefiere adoptar en países asiáticos, desvelan que, socialmente, se otorga un estatus social superior a quienes proceden de Asia al ser percibidos como "diferentes, pero no muy diferentes". Kubo (2010) y Sweeney (2013) señalan que la presión social por mantener la estratificación racial lleva a los padres a elegir el país de procedencia de sus hijos en función de los estereotipos atribuidos a las minorías étnicas y raciales.

En esta línea de investigación, Xing y Nakkula (2004) descubren que los padres tomaron la decisión de adoptar en China, porque la jerarquía racial les otorgaba una imagen positiva. La elección del país se convierte en una estrategia con la cual minimizar posibles problemas de adaptación e integración a través de una mayor semejanza fenotípica.

El propósito de este estudio es explorar el proceso de socialización cultural y racial de las familias adoptivas transraciales españolas en un contexto definido como de "paradoja transracial". En España, el fenómeno de las adopciones internacionales irrumpe en la década de 1990, veinte años después al vivido en otros países europeos. Sin embargo, su rápido incremento convirtió al país, en 2004, en el segundo del mundo en adopciones internacionales, registrándose en la fecha referida 5.541 adopciones en el extranjero. Desde entonces, el número de dichas adopciones ha dibujado una tendencia descendente tanto en España como a nivel global.

La notoriedad social del fenómeno de las adopciones internacionales confluye en el tiempo con el boom migratorio (Arango, 2010): entre 1997 y 2008, España es el país de la Unión Europea que recibió a más personas inmigrantes. La confluencia de ambos fenómenos ha dado paso a una línea de investigación interesada en abordar las experiencias raciales que comparten la población adoptada y la inmigrante. En España, la investigación señala que persisten las actitudes racistas y xenófobas basadas en las diferencias fenotípicas y que estas pueden llevar a etiquetar a las personas como "inmigrantes", a pesar de haber nacido en el país o tener la nacionalidad española (Cea D’Ancona y Valles, 2014). Por su parte, los estudios de San Román (2013) y San Román y Marre (2013) constatan que las familias españolas y los propios adoptados viven eventos raciales y de discriminación similares a la población inmigrante. 
Dos preguntas han guiado la investigación: ¿qué estrategias desarrollan para dar a conocer a sus hijos adoptivos la cultura del país de nacimiento? y ¿qué estrategias enseñan a sus hijos para afrontar posibles situaciones de discriminación, racismo o xenofobia? Para su desarrollo, asumimos las dos premisas que vinculan a los estudios emergentes sobre socialización cultural (Lee, 2003) y sociología de la etnicidad (Shiao et al., 2004: 5): las identidades raciales y étnicas son procesos en curso cuyo significado y contenido cambian a lo largo de la vida; y las familias, como agentes de socialización primaria, desempeñan un papel crucial en la gestión de la diferencia racial, étnica y cultural.

\section{La paradoja transracial y el proceso de socialización cultural y racial en familias adoptivas transraciales}

El modelo teórico de socialización cultural y racial en familias transraciales identifica tres dimensiones analíticas (Vonk, 2001; Massatti et al., 2004): competencia cultural y conciencia racial, o conocimiento de cómo opera la raza y la etnia en las vidas de las personas; planificación multicultural, o estrategias para enseñar a los niños transracialmente a conocer y participar en su cultura de nacimiento; y habilidades de afrontamiento, o competencia de los padres para preparar a sus hijos a enfrentarse a los estereotipos, prejuicios y racismos cotidianos. Las familias que son conscientes de la discriminación en la sociedad y de las ventajas y perjuicios que el racismo concede a quienes participan, o quedan excluidos, de la raza y la cultura dominante, afrontan la paradoja transracial con un trabajo deliberado por enseñar a sus hijos tanto su patrimonio cultural de nacimiento (enculturación) como herramientas de afrontamiento (racialización) (Lee, 2003; Lee et al., 2006).

Sin embargo, quienes no les otorgan relevancia desarrollan modelos de socialización basados en la asimilación cultural y reproduciendo el racismo daltónico, ideología racial contemporánea que se caracteriza por encubrir el discurso y las prácticas racistas, negar las desigualdades raciales presentes en la sociedad y permitir a la población blanca ${ }^{6}$ mantener su posición de privilegio sin parecer racista (Bonilla, 1999; Bonilla et al., 2004).

Los padres que participan en procesos de enculturación y racialización, contribuirán a la alfabetización racial de sus hijos, a través de la reflexión en 5 En este contexto, los términos de enculturación y de racialización remiten a los dos modelos de crianza (formas de socialización dentro de la familia) que Lee (2003) y Lee et al. (2006) han identificado como relevantes en las familias adoptivas transraciales.

6 La blancura actúa como una categoría social no marcada. 
el hogar de las cuestiones raciales y prácticas discriminatorias, privilegiando relaciones sociales con el grupo cultural y racial de referencia y formándoles mediante prácticas estéticas en el hogar y el consumo de arte, objetos materiales, música, juguetes y símbolos identificativos de su comunidad de origen (Twine, 2004); mientras que quienes despliegan un modelo de asimilación cultural y racismo daltónico minimizan los incidentes racistas, equiparándolos a otras diferencias identificables, y opinan que sus hijos serán considerados por sus méritos sin sopesar que la raza es un factor predictivo de oportunidades (Richardson, 2011).

En Europa, las investigaciones de Hübinette y Tigervall (2009) con adoptados coreanos en Suecia, De Grave (2015) con adoptados etíopes en Bélgica y las de Marre (2009; 2007), San Román (2013) y San Román y Marre (2013) en España, han destacado distintos aspectos de la paradoja transracial y del racismo daltónico imperante en las familias adoptivas transraciales. Sin embargo, se carece de investigación que arroje luz sobre el proceso de socialización cultural y racial en las familias adoptivas transraciales españolas.

\section{Metodología de la investigación}

El estudio que se presenta parte de los datos obtenidos en la encuesta "Las familias adoptivas y sus estilos de vida” (Rodríguez-Jaume y González-Río, 2014). La sección VII del cuestionario indagaba, específicamente, sobre las opiniones, actitudes y comportamientos de las familias adoptivas españolas en relación con distintas cuestiones asociadas con la familia transracial. La inexistencia de un registro de familias adoptivas (marco muestral), unido al hecho de que el fenómeno de las adopciones es un fenómeno en línea, motivó que el cuestionario se inscriba, específicamente, en las encuestas denominadas Computer Assisted Web Interviewing (CAWI).

En los meses que estuvo activada la encuesta (julio-septiembre de 2012), 835 madres y padres adoptivos accedieron al sitio web donde se alojó. Después de depurar los datos, se obtuvo una muestra no probabilística por autoselección de 230 padres que en 2012 contaban con una hija o hijo adoptado en el extranjero. El Cuadro $1^{7}$ resume sus características sociodemográficas así como las de sus hijas e hijos.

El proceso de socialización cultural y racial se exploró con base en los factores que la literatura especializada ha relacionado con las prácticas de socialización cultural:

7 El cuadro y las tablas se encuentran en el Anexo, al final del presente artículo. 
- Origen de las hijas e hijos adoptados agrupado por continentes. Lee et al. (2006) y Vonk et al. (2010) revelan que los padres que adoptaron en Europa son menos propensos a realizar prácticas culturales que los que lo hicieron en Asia y América Latina.

- Estructura familiar según tipo de filiación (adoptiva o mixta). Vonk y Massatti (2008) han mostrado que las familias sin hijos biológicos (filiación adoptiva) que adoptaron interracialmente se muestran más comprometidas con el proceso de socialización cultural. Sin embargo, la investigación realizada en España, mostró que las familias adoptivas españolas que cuentan con hijos biológicos (filiación mixta) perciben con mayor intensidad el estigma social de su modelo familiar (Rodríguez y Jareño, 2015).

- Años de convivencia con las hijas e hijos adoptados. Samuels (2009) identificó que los padres con mayor experiencia mostraban una ideología daltónica menor, probablemente porque a medida que su prole fue creciendo se enfrentaron a un mayor número de episodios racistas. ${ }^{8}$

Siguiendo la definición operativa que Vonk (2001) ofrece de competencia cultural de los padres adoptivos transraciales, y el análisis factorial que posteriormente realizaron Massatti et al. (2004) para testar su Transracial Adoption Parenting Scale (TAPS), en el cuestionario se incluyeron dos preguntas dirigidas a explorar la planificación multicultural que promueven las familias adoptivas. La primera pregunta indaga sobre las prácticas de socialización cultural con las que las madres y padres adoptivos exponen a sus hijos e hijas a contextos de socialización próximos a sus comunidades étnico-raciales de origen y a otras poblaciones diversas. Con este fin, el cuestionario incluyó lo siguiente:

Por favor, de las cuestiones que se relacionan a continuación, indique si usted las ha realizado (o realiza) o no las ha realizado (realiza):

1. Cambió su residencia a un barrio con características raciales o étnicas similares a las de su hijo/a adoptado/a.

2. Cambió de residencia para estar cerca de un colegio con características raciales o étnicas similares a las de su hijo/a adoptado/a.

3. Su ocio habitual incluye actividades y planes relacionados con la cultura del país de origen de su hijo/a adoptado.

4. Sus vacaciones anuales incluyen planes relacionados con la cultura del país de origen de su hijo/a adoptado/a.

8 Finalmente, esta variable se excluyó del análisis, pues $88,3 \%$ de la muestra indicó que el periodo de convivencia con sus hijas e hijos en el momento de la realización del estudio era de nueve meses. 
5. Procura seleccionar a sus amistades considerando las características culturales y/o étnicas de su hijo/a adoptado/a.

6. Procura seleccionar y preparar comida típica del país de origen de su hijo/a adoptado/a.

7. Lee libros sobre la cultura de su hijo/a adoptado/a.

8. Se ha iniciado o está profundizando en el idioma del país de origen de su hijo/a.

Cada uno de los ocho ítems se presentaba con alternativas de respuesta dicotómica $(1=$ sí y $2=$ no). La consistencia interna entre los ítems (Alfa de Cronbach) fue de 0,910 .

Una segunda pregunta profundizaba en la planificación y asiduidad de las actividades culturales. Con ella se buscaba determinar si el proceso de socialización cultural y racial expone a las niñas y niños adoptados a experiencias multirraciales y multiculturales. La pregunta incluida en el cuestionario se formuló como sigue:

Por favor, ¿podría indicar con qué frecuencia en su hogar...?:

1. Lee libros de autores africanos.

2. Cocina platos típicos del país de origen de su hijo/a.

3. Escucha músicas de artistas latinos.

4. Tiene arte asiático.

5. Ve TV que incluye programación del país de procedencia de su hijo/a.

6. Celebra las fiestas del país de origen de su hijo/a.

7. Invita a casa a adultos no españoles/as.

8. Invita a casa a niños/as no españoles/as.

Cada uno de los ítems se presentaba con tres alternativas de respuesta ( 1 =habitualmente; $2=$ algunas veces; $3=$ nunca). El nivel de consistencia interna de los ocho ítems (Alfa de Cronbach) fue de 0,777 con una puntuación media de 2,22 (SD=0,35).

Por último, para medir las estrategias de afrontamiento que las madres y padres enseñan a sus hijas e hijos en los hogares para superar episodios de discriminación racial o étnica, el cuestionario incluyó la pregunta siguiente:

Cuando su hijo/a adoptado/a ha vivido un episodio de racismo, discriminación o xenofobia (personalmente, en las noticias, en las películas,...), ¿con qué frecuencia usted o su entorno familiar...?:

1. Ha aprovechado para hablarle de la raza y el racismo.

2. Ha practicado posibles respuestas a comentarios insensibles, racistas, discriminatorios o xenófobos.

3. Demuestra tolerancia cero hacia comentarios insensibles, racistas, discriminatorios o xenófobos. 
4. Le enseña a demostrar sus preocupaciones y temores respecto a situaciones en las que se produzcan comentarios insensibles, racistas, discriminatorios o xenófobos.

5. Pide consejo a otros padres/madres que han podido pasar por la misma situación.

Cada uno de los ítems se presentaba con cinco alternativas de respuesta ( $1=$ nunca a $5=$ siempre). El nivel de consistencia interna de los cinco ítems (Alfa de Cronbach) fue de 1.

Por limitación de espacio, las tablas que se exhiben solo ofrecen una alternativa de respuesta. Para favorecer la comparabilidad, los porcentajes se calculan sobre el total de la población encuestada que respondió en cada una de las categorías de la variable mostrada y no sobre el conjunto de la muestra. En todo caso, se especifica la base muestral sobre la que se han determinado los indicadores. Los hallazgos que se presentan deben ser interpretados teniendo en consideración varias limitaciones metodológicas del estudio. La primera remite a la naturaleza no probabilística de la muestra que impide que los resultados puedan ser generalizados al conjunto de las familias que en España optaron por la adopción en el extranjero. En segundo lugar, el número reducido de casos registrados en algunas categorías de respuesta no permite identificar asociaciones causales y, en consecuencia, concretar posibles modelos explicativos del proceso de socialización cultural y racial objeto de estudio.

\section{Resultados y discusión}

\section{Prácticas de socialización cultural}

Un primer foco de atención analítico se dirigió a determinar si el proceso, en curso, de socialización cultural y racial de las niñas y niños adoptados en el extranjero por familias españolas les involucraba en la cultura y comunidad de origen. Este aspecto es especialmente relevante, dado que la investigación ha destacado que las prácticas de socialización cultural que implican inmersión en la comunidad de origen de las niñas y niños adoptados en el extranjero les provee de modelos de referencia (Vonk, 2001; Vonk et al., 2010), de una red de apoyo (Thomas y Tessler, 2007), mitiga el estigma percibido (Samuels, 2009), incrementa el orgullo de pertenencia a un grupo racial o minoría étnica, y desarrolla una identidad étnica positiva que favorece la gestión de episodios racistas y de discriminación (Vonk, 2001; Lee, 2003; Song y Lee, 2009; Lee et al., 2010). 
La Tabla $1^{9}$ aborda esta primera cuestión al recoger los valores porcentuales de cada una de las distintas prácticas de socialización cultural, que compromete a los hijos e hijas de las familias adoptivas españolas con la cultura y comunidad de origen. En la misma se han omitido las opciones "cambió su residencia a un barrio con características raciales o étnicas similares a las de su hijo/a adoptado/a", y "cambió de residencia para estar cerca de un colegio con características raciales o étnicas similares a las de su hijo/a adoptado/a", puesto que ninguna de ellas fue seleccionada por la muestra.

La ausencia de estas dos opciones de respuesta es, per se, significativa, al poner en evidencia que las instituciones extra-familiares y contextos sociales que las madres y padres adoptivos eligen como agentes de socialización secundaria de sus hijas e hijos no contemplan contacto con sus comunidades de origen, ni referentes que compensen la ausencia de recursos y experiencias raciales de los padres adoptivos. Reforzando este primer hallazgo, los datos revelan que el modo con el que los padres españoles adoptivos transmiten preferentemente a sus hijas e hijos los valores culturales, las creencias y las costumbres de su país de origen se produce a través de prácticas culturales que priorizan el vínculo formal (cognitivo) frente al experiencial que involucra directamente al niño. La única práctica mayoritariamente seleccionada fue la lectura de libros sobre la cultura del país de origen $(78,6 \%)$. Esta práctica, a su vez, es relevante entre quienes han adoptado en África (86\%) y Asia (82,5\%), y solo alcanza al 50\% de entre quienes lo hicieron en Europa. Incluso las "visitas de retorno", de creciente popularidad, ocupan una discreta presencia entre las actividades de socialización cultural y racial familiar.

En general, y salvo el aprendizaje del idioma, las familias españolas que adoptaron en el continente europeo son las que menos participan en prácticas de socialización cultural. Estos resultados son consistentes con los recogidos en los estudios comparados de Lee (2003) y Lee et al. (2006), en los cuales se aprecia que los padres que adoptaron en Rusia y en países de Europa del Este fueron quienes mostraron valores más bajos en las prácticas de enculturación y racialización, y, en consecuencia, los menos propensos a participar en actividades culturales ligadas al país de origen de sus hijas e hijos.

En el caso español, este patrón también ha sido identificado por Marre (2007) y San Román y Marre (2013), quienes han explicado que el menor compromiso de las familias españolas que adoptaron en Europa obedece a que sienten que la adopción se ha producido en "la misma cultura" (Marre, 2007: 82).

9 La Tabla 1 se encuentra en el Anexo, al final del presente artículo. 
Los datos reflejan que las familias que se configuran de forma interracial adoptando en América del Sur, África o Asia valoran, más que las que mantienen la homogeneidad racial, la importancia de proporcionar a su prole oportunidades, contextos y actividades culturales que promuevan su conciencia étnica, cultural y racial. Este hecho, unido a que la exposición y contacto con las comunidades de origen se produce más entre las familias españolas con filiación adoptiva mixta, sugiere que las que más se exponen en contextos de interacción social a la gestión de la "diferencia" mantienen una mayor predisposición a promover actividades culturales ligadas con los países de origen de sus hijas e hijos.

\section{Planificación multicultural}

El segundo ámbito de interés analítico se centró en describir las vías a través de las cuales los padres adoptivos enseñan a sus hijos adoptados a conocer, vincularse y participar en su cultura de nacimiento (Vonk, 2001: 251). Estas pueden ser numerosas y variar según el grado de contacto con el entorno real de la cultura de origen (Massatti et al., 2004: 44). Una menor participación implicaría facilitar el acceso a productos culturales específicos del país de origen; mientras que las rutas que favorecen un mayor contacto pueden incluir actividades que promueven el vínculo con la población que comparte la cultura de nacimiento del niño.

Twine (2004: 893) identificó dos estrategias de "resistencia" frente al racismo: la promoción de relaciones sociales con adultos y niños de la comunidad de origen, y las prácticas estéticas y de consumo en el hogar. Respecto a esta última, la etnógrafa describe cómo una minoría de los padres biológicos transraciales entrevistados seleccionaban cuidadosamente piezas de arte, objetos materiales, música, juguetes y símbolos con los que decoraban sus hogares y reproducían una cultura simbólica y estética antirracista, que promovía la identificación con los pueblos de África y las luchas de la comunidad negra.

En la Tabla 2 se recogen las respuestas ofrecidas sobre la frecuencia con la que las familias adoptivas españolas realizaban actividades culturales y consumían productos, ya fueran propios del lugar donde nacieron sus hijas e hijos o de otros lugares.

Los resultados obtenidos enfatizan la escasa presencia que, en la estrategia de socialización cultural y racial, alcanza el desarrollo de actividades y adquisición de productos culturales, con una puntuación media en la frecuencia de realización del conjunto de las actividades recogidas en la Tabla $2(\mathrm{M}=2,22)$, próxima a la opción de respuesta de "nunca" se lleva a cabo (3). Incluso la 
preparación de comida típica del país de origen, identificada como la segunda práctica de socialización, no se efectúa de forma cotidiana.

Cuando se reconoce la práctica habitual de las actividades, estas aparecen ligadas al país de origen de su prole. Así, la lectura de libros de autores africanos solo la realizan las familias que adoptaron en este continente $(2,8 \%)$; la música latina es una actividad mayoritaria entre las familias adoptivas en Latinoamérica (36\%), y el arte asiático está presente, fundamentalmente, en los hogares que adoptaron en Asia (35,7\%). Este dato sugiere que las familias que adoptaron en China son las más propensas a vincularse con la herencia étnica y cultural de sus hijos, a través del consumo de arte asiático.

Es probable, atendiendo a los hallazgos de la investigación etnográfica de Traver (2007: 206-207), que los objetos que se consumen se limiten a una reducida variedad de arte y que su función sea la de integrar en la vida familiar temas culturales y festividades chinas. Las actividades culturales se desarrollan, mayoritariamente, entre las familias adoptivas que cuentan con una filiación mixta. Estas, como han puesto de manifiesto estudios previos, perciben en mayor medida el estigma social que recae sobre las familias adoptivas (Rodríguez y Jareño, 2015), lo cual las puede predisponer a gestionar la discriminación racial, incrementando su planificación multicultural.

La exigua planificación multicultural puede responder a que los padres no perciben la necesidad de desplegar prácticas de socialización cultural, o bien, como ha sugerido Park (2012: 492-493), a que los padres ajustan sus esfuerzos de socialización a la demanda explícita de sus hijas e hijos cuando alcanzan la adolescencia. Si fuera así, este tipo de socialización se adscribiría a la estrategia de "elección del niño" que Lee (2003: 719) identificó, junto a la asimilación cultural, la enculturación y la inculcación racial, como modelos con los que las familias adoptivas afrontan la paradoja transracial. Esta tesis es difícil de cotejar con los datos disponibles, ya que la edad mediana de las hijas e hijos adoptados de las familias encuestadas se sitúa en los 7 años.

La reducida relevancia que las familias adoptivas otorgan a la planificación de actividades culturales contrasta con el valor que, en el ámbito de los deseos, le otorgan a la identidad bicultural. Así, el cuestionario aplicado recababa su opinión sobre si sus hijos e hijas "deben aprender nuestra cultura y costumbres, pero es bueno que también aprendan la cultura y costumbres de su país de procedencia”. La respuesta fue afirmativa para el $83,5 \%$ de la muestra, un porcentaje muy por encima al manifestado por la población española en el estudio de "Actitudes hacia la inmigración", donde el 36,9\% declaró que "aunque aprendan nuestra cultura y costumbres, es bueno que los inmigrantes mantengan su cultura y costumbres" (Centro de Investigaciones Sociológicas, 2012). 


\section{Estrategias de afrontamiento al racismo cotidiano}

El tercer foco de atención se centró en indagar en las estrategias de afrontamiento que en el proceso de socialización cultural y racial despliegan las familias adoptivas, con el fin de instruir y desarrollar habilidades entre sus hijos e hijas que les permitan gestionar tanto los racismos cotidianos, como las experiencias contradictorias a las cuales les expone la paradoja transracial. La Tabla 3 recoge los valores porcentuales alcanzados en cada una de las estrategias sobre las que fueron interrogados.

En este último apartado, nos enfocamos en aquellas que la investigación identifica como estrategias que contribuyen a afrontar activamente experiencias raciales, a saber (Vonk, 2001:251-252): enseñar a exteriorizar el racismo, validar los sentimientos de ira y dolor de los niños, transmitir el mensaje de que el racismo es injusto, evitar culpabilizar al niño, o prestar su ayuda en los casos que sus hijos les requieran para manejar una situación. Esto es, indagamos sobre lo que Twine (2004: 884), en el contexto de los padres biológicos interraciales, denominó como herramientas conceptuales que se enseñan en el hogar para responder al racismo.

Los datos muestran el reducido protagonismo de las estrategias de afrontamiento en el proceso de socialización cultural y racial de las familias adoptivas transraciales españolas, dado que su práctica oscila entre el 9,3\%, que "pide consejo a otros padres o madres que han podido pasar por la misma situación", y el 22,6\%, referido a "demostrar tolerancia cero hacia comentarios insensibles, racistas, discriminatorios o xenófobos”. Asimismo, el peso porcentual que asumen las distintas estrategias de afrontamiento sobre las que se les preguntó, desvela el disenso entre las familias españolas adoptivas entre el plano de los deseos y el de las realidades, pues si bien "demostrar tolerancia cero hacia comentarios insensibles, racistas, discriminatorios o xenófobos" y "enseñarles a demostrar sus preocupaciones y temores" frente a los mismos son las dos estrategias de afrontamiento más utilizadas (22,6\% y 20,4\%, respectivamente), la implicación activa de las madres y padres en la alfabetización racial de sus hijas e hijos, "practicando posibles respuestas" con las cuales afrontar estas situaciones, alcanza un reducido valor (15,1\%).

Estos hallazgos sugieren que las familias adoptivas españolas lejos de mirar su cotidianidad a través de un "lente crítico racial" (Twine, 2004), lo harían a través de un "lente daltónico" (Richardson, 2011) que les impide apreciar los efectos de las ventajas y perjuicios del racismo vigente en la sociedad y, en consecuencia, actuar para contrarrestarlo. En este sentido, y de acuerdo con Lee (2003), la orientación daltónica que adopta la estrategia 
de socialización desplegada por las familias adoptivas españolas transraciales se adscribiría a una estrategia de asimilación cultural.

Siguiendo la tesis de Lee et al. (2006), el bajo nivel de implicación de las madres y padres adoptivos encuestados puede reflejar una actitud inconsciente frente a la discriminación racial en la sociedad, lo cual les lleva a silenciar las cuestiones relativas a las identidades raciales (Willing y Fronek, 2014: 1141). Sin embargo, paradójicamente, las familias participantes en el estudio reconocieron el racismo imperante en la sociedad española. El cuestionario incluyó una escala de percepción del racismo en España que oscilaba entre (1) "nada racista” y (4) "muy racista". El valor medio obtenido fue 2,86.

Las diferencias que se observan en las estrategias de afrontamiento según origen pueden estar relacionadas con la percepción de los encuestados sobre el racismo en España. Así, los datos de la Tabla 3 sitúan las prácticas de afrontamiento en un continuo en donde las familias que cuentan con hijos procedentes de Latinoamérica, seguidas de las que adoptaron en África, son las que con mayor intensidad desarrollan habilidades de afrontamiento en sus hijas e hijos; mientras que las que lo hicieron en Europa se presentan como las menos comprometidas con estas prácticas. Este patrón se reproduce en la percepción que las familias encuestadas tienen del racismo en la sociedad española: el $80 \%$ y el 79,1\% de las familias adoptivas en Latinoamérica y África, respectivamente, consideran que la sociedad española es "bastante" o "muy" racista; en tanto, esta percepción es del 71,9\% y 69,8\% en las familias que adoptaron en Europa y Asia, respectivamente.

A diferencia de los resultados del estudio de Vonk y Massatti (2008), las estrategias de afrontamiento se practican más entre las familias con filiación adoptiva. Se precisa de investigación adicional que aborde esta posible relación con el proceso de socialización cultural y racial, así como la relevancia de la edad de sus hijos en la gestión de esta dimensión por parte de las madres y padres adoptivos.

\section{Conclusiones}

Este artículo nos acerca al proceso de socialización cultural y racial en las familias adoptivas transraciales, un aspecto escasamente abordado en España. Adicionalmente, los hallazgos obtenidos proporcionan evidencias empíricas que transcienden el ámbito de la sociología de las familias adoptivas, ya que permiten aproximarnos al estudio del racismo contemporáneo (racismo daltónico) y a las fronteras y jerarquías raciales en la sociedad española. 
En lo referente a las prácticas de socialización cultural, los resultados observados se corresponden con los obtenidos en investigaciones previas que revelaron que las prácticas de socialización cultural más comunes entre los padres adoptivos transraciales, lejos de implicar el contacto directo, práctico y prolongado con el medio cultural de la comunidad de origen de sus hijas e hijos, reproducen un vínculo formal y puntual con la cultura de nacimiento.

La escasa relevancia que adquieren entre las familias adoptivas españolas las prácticas de socialización cultural puede ser interpretada como un indicador del arraigo de la ideología daltónica en la sociedad. Esta impide que se aprecien las ventajas y perjuicios que el racismo concede a quienes participan, o quedan excluidos, de la raza y la cultura dominante.

El bajo racismo daltónico identificado en las familias encuestadas, unido a que las madres y los padres adoptivos no fueron ni serán objeto de estigmatización social y discriminación racial, pues no comparten con sus hijas e hijos su apariencia "extranjera", y a que, en general, disponen de un elevado estatus educativo, que les otorga una mayor confianza en su capacidad para transferir a sus hijas e hijos su propio éxito profesional y privilegios económicos (Ishizawa et al., 2006: 1216-1217; Samuels, 2009: 87), les puede llevar a relativizar el papel que desempeña la raza y la cultura en la vida de sus hijas e hijos, así como a reducir sus estrategias de socialización racial a prácticas testimoniales.

Por su parte, la planificación cultural en las familias adoptivas transraciales españolas en su cotidianidad es muy reducida (con un valor medio de 0,78 sobre 3 ). Esta aparece muy vinculada con el país de origen de su prole, $y$, nuevamente, compartir actividades que requieran interacciones con personas no españolas ocupa un lugar marginal. En este contexto, la planificación cultural lejos de desarrollar un modelo de socialización bicultural, como en el que se implica la población inmigrante (Thomas y Tessler, 2007), reproduciría una de las características centrales del mercado de la adopción y que Quiroz (2012: 528) ha denominado "turismo cultural" - acceso a productos, eventos y otras formas comercializadas de la "cultura"-.

Incluso las "visitas de retorno" no se asociarían tanto con una práctica de socialización cultural, sino, como han señalado Howell y Marre (2006:310), con el objetivo de disminuir el "fantasma" de lo desconocido de la "mochila" infantil que sus hijos traen desde sus países de origen.

Como corolario de lo expuesto, las familias encuestadas otorgan un escaso protagonismo a las estrategias de afrontamiento en el proceso de socialización cultural. A pesar de que manifiestan no tolerar comentarios racistas, discriminatorios o xenófobos, su implicación activa es reducida. No adoptar 
medidas que prepare a su prole a enfrentar situaciones racistas, estrategia central de la socialización racial, es el modelo de crianza que sucede, y reproduce, a la ideología daltónica (Richardson, 2011: 82). El "lente daltónico" de las familias adoptivas les impide apreciar la discriminación racial de la que son objeto las personas con diferencias fenotípicas y solo ven la necesidad de este aprendizaje cuando sus hijos, al ingresar en el ámbito escolar, viven situaciones de discriminación incuestionables (Massatti et al., 2004).

Los resultados presentados muestran una gestión ambivalente de las creencias y los comportamientos. Por un lado, el 83\% de los padres reconocen como deseable que sus hijas e hijos desarrollen una identidad bicultural, pero su planificación multicultural es exigua y prioriza el vínculo formal frente al experiencial. Por el otro, el 23\% declara manifestar su intolerancia frente a situaciones racistas, pero quienes practican respuestas activamente son el 15\% de la muestra. Esta ambivalencia puede estar reflejando una baja competencia cultural y racial de las madres y padres adoptivos.

En este contexto, las administraciones y organismos que colaboran en la gestión de la adopción internacional deberían incluir la competencia cultural y racial en los procesos selectivos y formativos de las familias españolas que desean crear una familia con un hijo adoptado en el extranjero, pues, de lo contrario, "navegará" en solitario en una sociedad que discrimina por raza, etnia y cultura (Samuels, 2009; Song y Lee, 2009; Sweeney, 2013).

Desde este contexto analítico, los datos sugieren que el grado de implicación de las familias adoptivas españolas en el proceso de socialización cultural y racial estaría relacionado con la jerarquía racial dominante. Esta dibujaría una línea imaginaria que sitúa, a un lado, a los colectivos que racial y étnicamente han sido construidos socialmente con una imagen positiva, ya sea porque comparten similitudes fenotípicas (adopción en Europa), o porque -como ha señalado Dorow (2006) - prevalece el modelo de discurso de minoría que reconstruye el estereotipo que presenta a las personas adoptadas en Asia como una población inteligente, trabajadora y más asimilable que otras minorías.

Esta imagen positiva actúa como factor de protección, reduciendo las opciones de enfrentamiento a situaciones de discriminación racial y étnica, mitigando la percepción del carácter racista de la población española y, en consecuencia, disuadiendo a los padres de la necesidad de transmitir este aprendizaje. Al otro lado de la línea que imaginariamente traza los límites sociales de la jerarquía racial en la sociedad española, se ubicarían las minorías que gozan de menor prestigio social y que en esta investigación aparecerían representadas por las niñas y niños procedentes de América Latina y 
África. En este caso, tanto las evidentes diferencias fenotípicas (adopción en África), como los prejuicios sociales negativos con los que se vincula a la población latinoamericana (asociación en pandillas, vinculación con drogas y abandono escolar), pueden exponer en mayor medida a las familias a situaciones de discriminación racial, a la percepción de que su entorno es racista $y$, en consecuencia, a manifestar un mayor compromiso con el proceso de socialización cultural.

Por último, estas evidencias sugieren que el dominio en España de los flujos de adopción internacional procedente de Asia y Europa obedece, entre otras causas, a la presión social a la que son sometidas las familias adoptivas por mantener la estratificación racial hegemónica, y que llevaría a las madres y padres a elegir el país de procedencia en función de los estereotipos atribuidos a las minorías raciales y étnicas. Esta tesis, avalada para el contexto norteamericano (Kubo, 2010; Sweeney, 2013), plantea una futura línea de investigación para el caso de las adopciones internacionales en España.

\section{Referencias}

Arango, Joaquín (2010), "Después del gran boom: la inmigración en la bisagra del cambio", en Aja, Eliseo et al. [eds.], La inmigración en tiempos de crisis. Anuario de la inmigración en España (edición 2009), España: Barcelona Center for International Affairs). Disponible en: https://www.cidob.org/es/publicaciones/serie_de_publicacion/ anuario_cidob_de_la_inmigracion/la_inmigracion_en_tiempos_de_crisis_anuario_ de_la_inmigracion_en_espana_edicion_2009 [24 de septiembre de 2018].

Balibar, Etienne (1991), “¿Existe un neorracismo?”, en Wallerstein, Immanuel y Etienne Balibar, Raza, nación y clase, Madrid: IEPALA.

Bonilla-Silva, Eduardo (1999), "The essential social fact of race", en American Sociological Review, vol. 64, núm. 6. DOI: 10.2307/2657410. Disponible en: https://doi. org/10.2307/2657410 [7 de febrero de 2018].

Bonilla-Silva et al. (2004), "I did not get that job because of a black man...': The story lines and testimonies of color-blind racism", en Sociological Forum, vol. 19, núm. 4. DOI: 10.1007/s11206-004-0696-3. Disponible en: https://doi.org/10.1007/s11206-0040696-3 [ 4 de febrero de 2018].

Cea d'Ancona, $\mathrm{M}^{\mathrm{a}}$ Ángeles y Valles, Miguel (2014), Evolución del racismo y la xenofobia en España, Informe-Encuesta 2012, España: Observatorio Español del Racismo y la Xenofobia y Ministerio de Trabajo e Inmigración.

Centro de Investigaciones Sociológicas (2012), "Actitudes hacia la inmigración (VI)", en Encuesta Estudio, núm. 2967, España: Centro de Investigaciones Sociológicas. Disponible en: http://www.cis.es/cis/opencm/ES/1_encuestas/estudios/ver. jsp?estudio $=13244$ [ 4 de febrero de 2018]. 
De Grave, Katrien (2015), “They have our culture': negotiating migration in BelgianEthiopian transnational adoption", en Ethnos: Jo71-90urnal of Anthropology, vol. 80, núm. 1. DOI: 10.1080/00141844.2013.813565. Disponible en: https://doi.org/10.1 080/00141844.2013.813565 [4 de febrero de 2018].

Dorow, Sara (2006), Transnational adoption: a cultural economy of race, gender, and kinship, Estados Unidos: University Press.

Howell, Signe y Marre, Diana (2006), “To kin a transnationally adopted child in Norway and Spain: the achievement of resemblances and belonging", en Ethnos: Journal of Anthropology, vol. 71, núm. 3. DOI: 10.1080/00141840600902679 Disponible en: https://doi.org/10.1080/00141840600902679 [5 de marzo de 2018].

Hübinette, Tobias y Tigervall, Carina (2009), "When racism becomes individualised: writing about experiences of racialisation among adult adoptees and adoptive parents of Sweden”, en Keskinen, Suvi et al. [eds.], Complying With Colonialism: Gender, Race and Ethnicity in the Nordic Region, Inglaterra: Ashgate.

Ishizawa, Hiromi et al. (2006), "Constructing interracial families through intercountry adoption”, en Social Science Quarterly, vol. 87, núm. 5. DOI: 10.1111/j.15406237.2006.00424.x. Disponible en: https://doi.org/10.1111/j.15406237.2006.00424.x [7 de febrero de 2018].

Kubo, Kazuyo (2010), "Desirable difference: the shadow of racial stereotypes in creating transracial families through transnational adoption", en Sociology Compass, vol. 4, núm. 4. DOI: https://doi.org/10.1111/j.1751-9020.2010.00274.x. Disponible en: https:// doi.org/10.1111/j.1751-9020.2010.00274.x [26 de enero de 2018].

Lee, Richard (2003), "The transracial adoption paradox: history, research, and counseling implications of cultural socialization", en Counseling Psychologist, vol. 31, núm. 6. DOI: 10.1177/0011000003258087. Disponible en: https://doi. org/10.1177/0011000003258087 [2 de febrero de 2018].

Lee, Richard et al. (2010), "Comparing the ethnic identity and well-being of adopted Korean Americans with immigrant/U.S.-born Korean Americans and Korean International Students", en Adoption Quarterly, vol.31, núm.6.DOI: 10.1080/10926751003704408. Disponible en: https://doi.org/10.1080/10926751003704408 [24 de enero de 2018].

Lee, Richard et al. (2006), "Cultural socialization in families with internationally adopted children”, en Journal of Family Psychology, vol. 31, núm. 6. DOI: 10.1037/08933200.20.4.571. Disponible en: https://doi.org/10.1037/0893-3200.20.4.571 [24 de enero de 2018].

Marre, Diana (2007), "I want her to learn her language and maintain her culture'. Transnational adoptive families' views of 'cultural origins", en Wade, Peter [ed.], Race, ethnicity and nation: perspectives from kinship and genetics. Estados Unidos-Inglaterra: Berghahn Books.

Marre, Diana (2009), "We do not have immigrant children at this school, we just have children adopted from abroad': flexible understandings of children's 'origins”, en Briggs, Laura [ed.], International Adoption Global Inequalities and the Circulation of Children, Estados Unidos-Inglaterra: New York University Press. DOI: 10.18574/ nyu/9780814791011.003.0013. Disponible en: https://doi.org/10.18574/ nyu/9780814791011.003.0013 [7 de febrero de 2018].

Massatti, Richard et al. (2004), "Reliability and validity of the transracial adoption parenting scale", en Research on Social Work Practice, vol. 14, 
María José Rodríguez-Jaume y María José González-Río. Socialización cultural y racial en las familias españolas adoptivas transraciales

núm. 1. DOI: 10.1177/1049731503257807. Disponible en: https://doi. org/10.1177/1049731503257807 [3 de febrero de 2018].

Park, Soyeon (2012), “Caucasian parents' experience with transnational-transracial adoption: a phenomenological study", en International Journal of Child, Youth and Family Studies, vol. 4, núm. 1. DOI: 10.18357/ijcyfs34.1201211553. Disponible en: https://doi.org/10.18357/ijcyfs34.1201211553 [26 de enero de 2018].

Quiroz, Pamela Anne (2012), "Cultural tourism in transnational adoption: 'staged authenticity' and its implications for adopted children”, en Journal of Family Issues, vol. 33, núm. 4. DOI: 10.1177/0192513X11418179. Disponible en: https://doi. org/10.1177/0192513X11418179 [3 de febrero de 2018].

Rodríguez-Jaume, María José (2012), Encuesta "Las familias adoptivas y sus estilos de vida" (FAMADOP, 2012), en El (baby) boom de las adopciones internacionales en España. Una investigación sociológica sobre las familias adoptivas y sus estilos de vida (I+D+I-2008-2011), España: Ministerio de Economía y Competitividad.

Rodríguez-Jaume, María José y González-Río, María José (2014), “Las encuestas autoadministradas por Internet. Un estudio de caso: las familias adoptivas y sus estilos de vida”, en Empiria. Revista de Metodología de Ciencias Sociales, núm. 29. DOI: 10.5944/empiria.29.2014.12944. Disponible en: https://doi.org/10.5944/ empiria.29.2014.12944 [4 de febrero de 2018].

Rodríguez, María José y Jareño, Diana (2015), "Estigma social y adopción internacional en España: ¿es la familia adoptiva un modelo familiar menos 'auténtico' que los basados en lazos biológicos”, en Papers: Revista de Sociología, vol. 100, núm. 2. DOI: 10.5565/rev/ papers.2070. Disponible en: https://doi.org/10.5565/rev/papers.2070 [4 de febrero de 2018].

Richardson, Christine (2011), “Transracial adoption: promoting racial literacy or perpetuating colorblind racism?”, en McNair Scholars Research Journal, año 7, núm. 1, Estados Unidos: Boise State University. Disponible en: http://scholarworks. boisestate.edu/mcnair_journal/vol7/iss1/14/ [4 de febrero de 2018].

Samuels, Gina Miranda (2009), "Being raised by white people': navigating racial difference among adopted multiracial adults", en Journal of Marriage and Family, núm. 71. DOI: 10.1111/j.1741-3737.2008.00581.x Disponible en: https://doi.org/10.1111/j.17413737.2008.00581.x [3 de febrero de 2018].

San Román, Beatriz (2013), “'I am White ... even if I am racially black' 'I am Afro-Spanish': confronting belonging paradoxes in transracial adoption”, en Journal of Intercultural Studies, vol. 32, núm. 3. DOI: 10.1080/07256868.2013.787400. Disponible en: https://doi.org/10.1080/07256868.2013.787400 [ 4 de febrero de 2018].

San Román, Beatriz y Marre, Diana (2013), “De 'chocolatinas' y 'princesas de ojos rasgados': sobre la diferencia 'fisonómica' en la adopción transracial en España”, en López Matheu, Carmen et al. [eds.], Maternidades, procreación y crianza en transformación, España: Bellaterra.

Shiao Jiannbin et al. (2004), "Shifting the spotlight: exploring race and culture in KoreanWhite adoptive families", en Race and Society, vol. 7, núm. 1. DOI: 10.1016/j. racsoc.2004.11.003. Disponible en: https://doi.org/10.1016/j.racsoc.2004.11.003 [26 de febrero de 2018].

Song, Sueyoung y Lee, Richard (2009), "The past and present cultural experiences of adopted Korean American adults", en Adoption Quarterly, vol. 12, núm. 
Convergencia Revista de Ciencias Sociales, núm. 80, 2019, Universidad Autónoma del Estado de México

1. DOI: 10.1080/10926750902791946. Disponible en: https://doi. org/10.1080/10926750902791946 [26 de febrero de 2018].

Sweeney, Kathryn (2013), "Race-conscious adoption choices, multiraciality, and colorblind racial ideology”, en Family Relations. DOI: 10.1111/j.1741-3729.2012.00757.x. Disponible en: https://doi.org/10.1111/j.1741-3729.2012.00757.x [28 de enero de 2018].

Thomas, Kristy y Tessler, Richard (2007), "Bicultural socialization among adoptive families. Where there is a will, there is a way", en Journal of Families Issues, vol. 28, núm. 9. DOI: 10.1177/0192513X07301115. Disponible en: https://doi. org/10.1177/0192513X07301115 [26 de febrero de 2018].

Traver, Amy (2007), “Home (land) Décor: China Adoptive Parents' Consumption of Chinese Cultural Objects for Display in their Homes", en Qualitative Sociology, vol. 30, núm. 3. DOI: 10.1007/s11133-007-9068-7. Disponible en: https://doi. org/10.1007/s11133-007-9068-7 [4 de febrero de 2018].

Twine, France (2004), "A white side of black Britain: The concept of racial literacy", en Ethnic and Racial Studies, vol. 27, núm. 6. DOI: 10.1080/0141987042000268512. Disponible en: https://doi.org/10.1080/0141987042000268512 [4 de febrero de 2018].

Vonk, Elizabeth (2001), "Cultural competence for transracial adoptive parents", en Social Work, vol. 46, núm. 3. DOI: 10.1093/sw/46.3.246. Disponible en: https://doi. org/10.1093/sw/46.3.246 [26 de febrero de 2018].

Vonk, Elizabeth y Massatti, Richard (2008), "Factors related to transracial adoptive parents' levels of cultural competence", en Adoption Quarterly, vol. 13, núm. 3. DOI: Disponible en: $10.1080 / 10926750802421966$. https://doi.org/10.1080/10926750802421966 [3 de febrero de 2018].

Vonk, Elizabeth et al. (2010), "Cultural socialization practices in domestic and international transracial adoption", en Adoption Quarterly, núm. 13. DOI: 10.1080/10926755.2010.524875. Disponible en: https://doi.org/10.1080/1092675 5.2010.524875 [3 de febrero de 2018].

Willing, Indigo y Fronek, Patricia (2014), "Constructing identities and issues of race in transnational adoption: the experiences of adoptive parents", en British Journal of Social Work, vol. 44, núm. 5. DOI: 10.1093/bjsw/bcs171. Disponible en: https://doi. org/10.1093/bjsw/bcs 171 [3 de febrero de 2018].

Xing, Tony y Nakkula, Michael (2004), "White parents' attitudes towards their adopted chinese daughters' ethnic identity”, en Adoption Quarterly, núm. 7. DOI: 10.1300/ J145v07n04_03. Disponible en: https://doi.org/10.1300/J145v07n04_03 [28 de enero de 2018].

Yngvesson, Barbara (2000), "Un niño de cualquier color': race and nation in intercountry adoption”, en Jenson, Jane y Boaventura de Sousa Santos [eds.], Globalizing institutions: case studies in regulation and innovation, Inglaterra: Ashgate. 


\section{Anexo}

\section{Cuadro 1}

Características demográficas de la muestra de familias adoptivas transraciales españolas (2012)

\begin{tabular}{|c|c|c|}
\hline Características & $\mathrm{N}$ & $\%$ \\
\hline Sexo encuestado/a & $\begin{array}{c}192 \\
38\end{array}$ & $\begin{array}{l}83,5 \\
16,5\end{array}$ \\
\hline Edad encuestado/a $($ media $=44,9)$ & $\begin{array}{c}42 \\
149 \\
37 \\
2 \\
\end{array}$ & $\begin{array}{c}18,3 \\
64,8 \\
16,1 \\
0,9\end{array}$ \\
\hline $\begin{array}{r}\text { Soltero/a } \\
\text { Casado/a } \\
\text { Viudo/a } \\
\text { Separado/a } \\
\text { Cohabitación } \\
\text { Con pareja sin convivencia }\end{array}$ & $\begin{array}{c}23 \\
192 \\
1 \\
9 \\
3 \\
2 \\
\end{array}$ & $\begin{array}{r}10,0 \\
83,5 \\
0,4 \\
3,9 \\
1,3 \\
0,9\end{array}$ \\
\hline Nivel de estudios encuestado/a & $\begin{array}{l}23 \\
40 \\
167\end{array}$ & $\begin{array}{l}9,9 \\
17,4 \\
72,7\end{array}$ \\
\hline $\begin{array}{l}\text { Estructura familiar según tipo de filiación }{ }^{1} \\
\text { Filiación adoptiva } \\
\text { Filiación mixta }\end{array}$ & $\begin{array}{c}176 \\
54\end{array}$ & $\begin{array}{l}76,5 \\
23,5 \\
\end{array}$ \\
\hline Sexo hijo/a adoptado/a & $\begin{array}{l}184 \\
124 \\
\end{array}$ & $\begin{array}{l}59,7 \\
40,3 \\
\end{array}$ \\
\hline Años de convivencia $(\text { media }=5,3)^{2}$ & $\begin{array}{c}137 \\
66 \\
21 \\
2\end{array}$ & $\begin{array}{c}59,6 \\
28,7 \\
9,1 \\
1,7\end{array}$ \\
\hline $\begin{array}{r}\text { Europa } \\
\text { Africa } \\
\text { América del Sur } \\
\text { Asia }\end{array}$ & $\begin{array}{c}39 \\
60 \\
34 \\
156\end{array}$ & $\begin{array}{c}13,5 \\
20,8 \\
11,8 \\
54\end{array}$ \\
\hline
\end{tabular}

Fuente: Elaboración propia a partir de los datos de la encuesta "Las familias adoptivas y sus estilos de vida" (Rodríguez-Jaume, 2012).

Notas: 1. Filiación adoptiva: solo hijos/as adoptivos/as. Filiación mixta incluye: hijos/as biológicos/as y adoptivos/as (22,6\%) e hijastros/as e hijos/as adoptivos/as (0,9\%). 2. Años de convivencia, se ha calculado tomando como referencia el primer hijo/a adoptado/a. 3. Las adopciones en Europa procedieron en 38\% de Rusia (15), en 28,2\% de Kazajstan (11) y en $26 \%$ de Ucrania (10); las de África se produjeron en 73\% en Etiopía (44); mientras que las de Asia se centraron en $85 \%$ en China (132). 


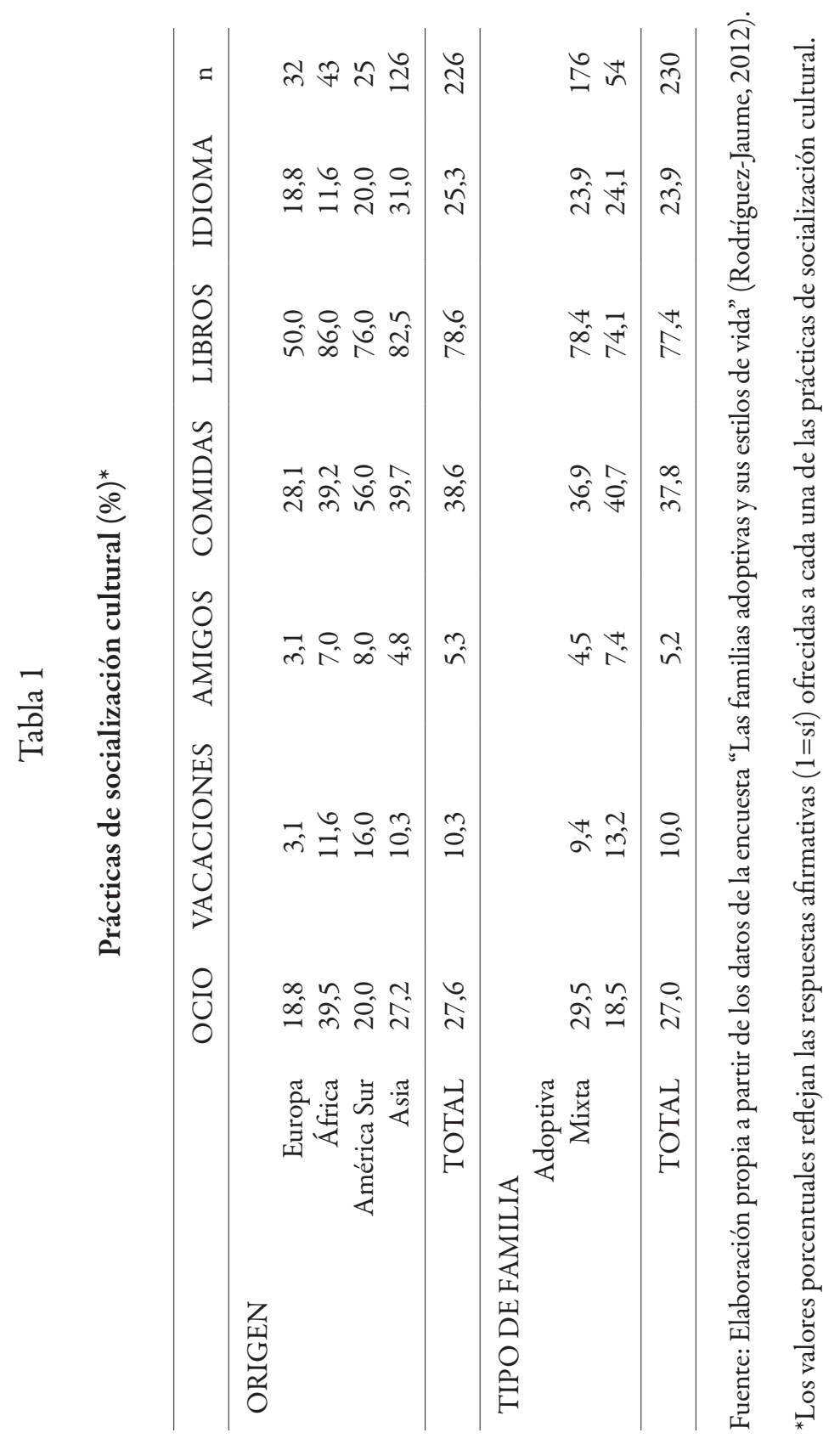


María José Rodríguez-Jaume y María José González-Río.

Socialización cultural y racial en las familias españolas adoptivas transraciales

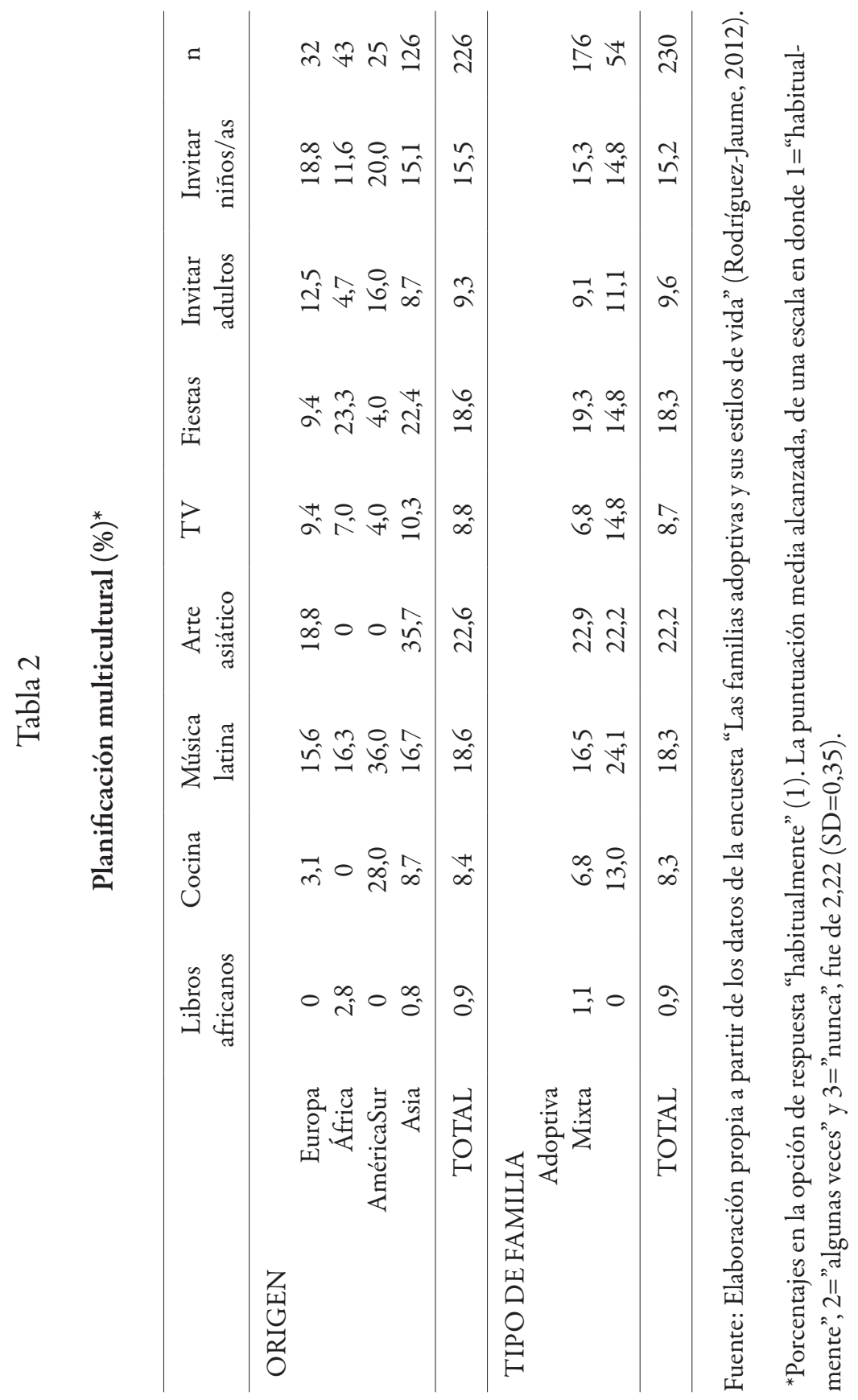




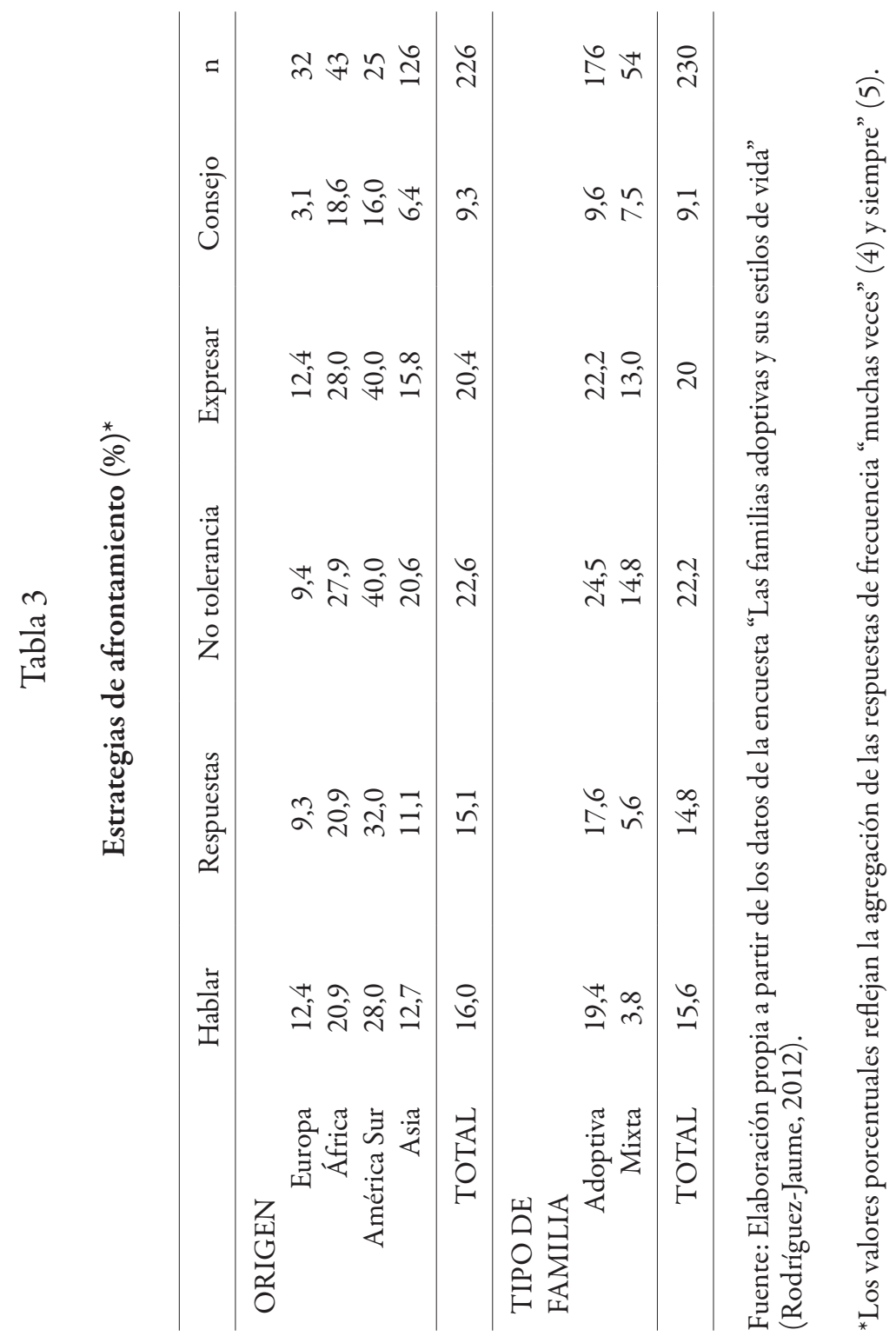


María José Rodríguez-Jaume. Doctora en Sociología. Universidad de Alicante (UA). Departamento de Sociología I. Líneas de investigación: sociología de las familias, sociología de las adopciones y estudios de género. Publicaciones recientes: Rodríguez-Jaume, María José (2018/2019), "El 'nuevo racismo' desde la lente de la migración silenciosa: la adopción interracial en España”, en Migraciones Internacionales (en prensa); Rodríguez-Jaume, María José y Garrigós, J. I. (2017), Análisis sociológico con documentos personales, Madrid: Centro de Investigaciones Sociológicas; Rodríguez-Jaume, María José y Jareño, D. (2015), "Estigma social y adopción internacional en España: ¿es la familia adoptiva un modelo familiar menos 'auténtico' que los basados en lazos biológicos?, en Papers, núm. 100, DOI: 10.5565/rev/papers. 2070.

María José González-Río. Doctora en Sociología. Universidad de Alicante (UA). Departamento de Sociología II. Líneas de investigación: sociología de las adopciones, estudios de género, comunicación y técnicas de investigación. Publicaciones recientes: Rodríguez Díaz, Ma R. y González-Río, María José (2016), "La Web 2.0 herramienta de empoderamiento de las empresarias pymes. Un estudio de caso en el contexto andaluz", en Feminismos, núm. 27, DOI: 10.14198/fem.2016.27.12; Espinar-Ruiz, E. y González-Río, María José (2015), "Uso de Internet y prácticas políticas de los jóvenes españoles", en Convergencia Revista de Ciencias Sociales, núm. 69, disponible en: http:// convergencia.uaemex.mx/article/view/3632; Rodríguez, María José y González-Río, María José (2014), "Las encuestas autoadministradas por internet. Un estudio de caso: las familias adoptivas y sus estilos de vida”, en Empiria: Revista de Metodología de Ciencias Sociales, núm. 29, DOI: 10.5944/empiria.29.2014.12944. 